\title{
Généalogie des modèles de la libéralisation
}

Jean-Marc BOUSSARD

Chercheur associé à I'INRA,

Membre de l'Académie d'Agriculture de France

$<$ <marc.boussard@wanadoo.fr>

\begin{abstract}
Many econometric models are providing a scientific guarantee in support of full trade liberalisation. This is an old tradition, as old as the $18^{\text {th }}$ century "physiocrates" (those who wanted a "natural law" governing economy. Most of these models, however, are not younger than Leon Walras's. The latter is a famous economist of the $19^{\text {th }}$ century, one of the first having introduced the idea of a general equilibrium model. After more than one century, and many experiences, the question arises of the validity of Leon Walras's intuition, so genial they may have been at the time. A modern analysis of the liberalisation benefits should now rely on dynamic models, in which uncertainty regarding futures prices is explicitly taken in account. With such models, it would be possible to evaluate both the benefits arising from a better world resources allocation and the cost associated with the removal of many regulation policies which, historically, had been created under the pressure of market failures, and which a total liberalisation would make impossible to continue.
\end{abstract}

Key words: CGE, Walras, liberalisation, risk, econometric models

budget. Mais le problème central de l'économie n'est pas là : il consiste à évaluer les effets globaux de ces décisions individuelles, dont rien n'assure a priori qu'elles sont compatibles entre elles, ou qu'elles ne s'annulent pas réciproquement. De ce point de vue, certains analystes, à ces époques, étaient bien conscients de ce que telle ou telle action du Prince pouvait avoir des résultats plus ou moins bénéfiques ou dommageables sur tel ou tel aspect de la vie économique : ainsi existe-il d'assez nombreux textes très anciens - dont certain de très grande qualité - sur les conséquences de l'altération des monnaies (incorporation de cuivre ou de plomb dans les pièces $d^{\prime}$ 'or). Mais leurs auteurs avaient rarement une vision d'ensemble des mécanismes économiques, depuis le comportement des individus jusqu'au déterminisme de la « richesse des nations».

Une telle vision s'est progressivement formée aux XVII et au XVIII ${ }^{\mathrm{e}}$ siècles, en particulier en France et en Angleterre. Pour comprendre comment, il faut probablement partir de Colbert. Celui-ci avait élaboré une doctrine dans laquelle il appliquait aux États la notion de richesse qui a cours dans les affaires privées: plus on a d'or, plus on est riche. Contrairement à l'Espagne - la puissance mondiale de l'époque - la France n'avait pas d'or. Pour s'en procurer, il fallait donc développer les productions «réelles» de biens agricoles et industriels, tout en essayant de vendre à l'étranger sans rien lui acheter - seul moyen d'accumuler l'or dans le trésor royal. Pour cela, ce dernier devait se faire industriel, créant des manufactures et des fermes-écoles.

Pour cette dernière raison, Colbert est souvent crédité - avec sans doute un peu d'exagération - de l'invention de l'économie dirigée. De ce point de vue, son action fut plutôt positive : elle entraîna un développement sensible des activités économiques en France, contrairement à ce qui se passait en Espagne, assise sur son tas d'or péruvien et déclinant rapidement. Mais sa manœuvre pour accumuler de l'or dans les caisses publiques échoua largement car les dépenses furent souvent plus grandes que les recettes. C'était du reste souhaitable car, évidemment, il n'y a aucune utilité à accumuler des stocks d'un produit sans intérêt direct comme l'or. En revanche, la dilapidation des stocks d'or pour financer les guerres de Louis XIV, si elle n'a sans doute pas causé le bonheur du peuple, a du moins apporté de grandes satisfactions au souverain et à son entourage, ce qui, après tout, est mieux que rien. Ce qui est surprenant, c'est que les contemporains n'ont jamais été conscients de ces phénomènes, en l'absence de toute comptabilité nationale et de tout repère théorique.

Si les règles établies par Colbert furent critiquées, ce n'est pas d'abord à cause de la méconnaissance de la logique des flux réels et financiers qui sous-tendait son analyse, et qui rendait ces politiques irréalisables à long terme en l'absence du gaspillage de biens de consommation associé à la guerre. Les premières attaques contre le colbertisme portèrent sur l'aspect « économie dirigée », et les absurdités 
bureaucratiques auxquelles il donnait lieu. Bien souvent, les bureaux refusaient des actions évidemment utiles, ou en promouvaient d'autres visiblement absurdes. Contre ces dérives, il apparaissait possible de compter sur l'intérêt bien compris des marchands, qui, en recherchant le profit, feraient tous leurs efforts pour apporter le maximum de marchandises là où on en avait besoin, pour le plus grand bonheur de tous.

C'est ainsi que les Physiocrates (ceux qui veulent gouverner conformément à Physis, la nature) théorisent alors le libre-échange et les avantages à tirer du bon fonctionnement du marché 1 . Même si les théories libérales des Physiocrates étaient passablement naïves, elles ont fait grandement avancer la réflexion économique. En particulier, ils ont créé la comptabilité nationale, à la base de tous les modèles dont il va être question plus loin ${ }^{2}$. C'est en partant de leurs intuitions qu'une génération plus tard, en Angleterre, Adam Smith devient le "père" de la science économique. II n'est sûrement pas le premier à avoir imaginé que la poursuite égoïste de l'intérêt individuel pouvait déboucher sur le bien-être collectif, mais il est le premier à avoir présenté une vision d'ensemble de la « richesse des nations ». Après lui, de nombreux auteurs, au XIX ${ }^{\mathrm{e}}$ siècle, ont creusé et formalisé ses analyses - en particulier David Ricardo et Robert Malthus.

Il est vite apparu que la rigueur du raisonnement serait puissamment aidée par l'usage de « modèles » mathématiques - des systèmes d'équations dont les variables se comportent plus ou moins comme le feraient, le cas échéant, les quantités économiques correspondantes. L'avantage évident d'une telle démarche est d'utiliser la rigueur du raisonnement mathématique pour baliser le chemin qui va des prémisses aux conclusions. Ceci est spécialement utile lorsque sont en jeu de nombreuses variables en interaction les unes avec les autres. Accessoirement, si l'on dispose de données ad

\footnotetext{
${ }^{1}$ En l'espèce, ils n'avaient pas complètement raison. Les politiques «libérales» de Turgot (qui tenta d'abolir les obstacles à la libre circulation des grains, le marché devant assurer tout seul l'approvisionnement des provinces en déficit de production) dans les années 1780 ne donnèrent pas toujours les résultats escomptés. À plusieurs reprises, il fut obligé de rétablir sous l'empire de la nécessité le régime des autorisations de transport qui avait été mis en place progressivement par les régimes successifs depuis Louis XIV. De plus, par les craintes qu'elles engendrèrent, les tentatives de libéralisation de Turgot comptent parmi les causes de la Révolution française.

${ }^{2}$ Le Tableau économique de Quesnay constitue la première tentative pour représenter de façon cohérente, à partir de données réelles, l'ensemble des flux de paiements d'un pays comme la France.
}

hoc, la formalisation mathématique permet de comparer à la réalité les prévisions d'un modèle, et donc, d'en apprécier la valeur.

Cette idée de l'utilisation des mathématiques dans les sciences sociales a germé au XIX ${ }^{\mathrm{E}}$ siècle sous la plume de divers auteurs, y compris Karl Marx ${ }^{3}$. En particulier, pour ce qui concerne la « richesse des nations", c'est en cherchant à exprimer sous forme mathématique les discours d'Adam Smith et de ses successeurs que Léon Walras est devenu à l'origine de la grandiose vision mathématique de «l'équilibre général ». II faut dire un mot de son modèle, qui reste l'archétype de tous les autres.

\section{Le modèle de Walras et la théorie de l'équilibre général}

Dans la ligne de pensée des Physiocrates, mais en en perfectionnant l'analyse et en l'exprimant sous forme mathématique, Walras part de quatre hypothèses fondamentales :

1) Tous les producteurs maximisent leur profit, sous la contrainte des limitations imposées par les techniques et la disponibilité en facteurs de production (stricto sensu, non susceptibles d'être produits, tels que terre, ou travail, ou "biens intermédiaires", issus d'autres industries, comme les engrais)

2) Tous les consommateurs maximisent leur « utilité » sous contrainte de budget.

3) Les budgets des consommateurs sont déterminés par les droits qu'ils détiennent sur les facteurs de production (tous les hommes ont des droits sur leur travail, et reçoivent donc un salaire en proportion de la quantité de travail qu'ils fournissent ; certains ont des droits sur la terre, et reçoivent une rente proportionnelle au nombre d'hectares qu'ils possèdent, etc.).

4) les prix équilibrent l'offre et la demande sur tous les marchés, qu'il s'agisse des biens de consommation ou des facteurs de production donnés en quantité fixe.

Alors, si I'on connaît une expression algébrique pour les fonctions d'utilité (qui exprime comment l'utilité du consommateur dépend des quantités de biens qu'il consomme) et pour les fonctions de production (qui expriment quelle quantité de produits il est possible d'obtenir à partir d'une collection donnée d'« inputs »), il facile de montrer que l'écriture de ces quatre conditions procure exactement autant d'équations que d'inconnues (essentiellement, les

\footnotetext{
${ }^{3}$ Ce qui ne manque pas de sel, si l'on songe que les " Marxistes » des années 1970 en France étaient en général farouchement opposés à toute formalisation mathématique.
}

quantités produites et les prix), sauf une. L'équation manquante correspond à la quantité de monnaie en circulation, qui est arbitraire, de sorte que les prix sont fixés à un coefficient de proportionnalité près.

Walras croyait que le fait qu'il y ait autant $d^{\prime}$ équations que $d$ 'inconnues était suffisant pour que la solution existe et soit unique. II $\mathrm{n}^{\prime}$ en est rien, et beaucoup d'autres conditions sont nécessaires pour cela. En même temps, on peut imaginer sur l'équilibre des théorèmes qui ne reposent pas sur l'existence de fonctions de production ou de fonctions d'utilité algébriques, mais plutôt sur des propriétés topologiques des préférences, ou de l'ensemble des productions réalisables dans un espace multidimensionnel, comme la « convexité ». Cela permet de généraliser la notion, et d'éviter que l'existence de l'équilibre ne dépende d'hypothèses assez restrictives comme l'existence de formules analytiques pour les fonctions de production.

En plus, il est alors possible de montrer que toute solution d'un tel système présente des propriétés d'optimalité très séduisantes : il est impossible d'accroître le bien-être d'un consommateur sans diminuer celui d'un autre, de sorte que même si la répartition des revenus est « injuste ", il est cependant certain que l'on tire le meilleur des ressources disponibles. On peut d'ailleurs modifier arbitrairement la répartition des revenus, en modifiant la nature des droits des individus sur les facteurs de production. On pourrait ainsi en particulier attribuer au travail une partie des revenus du capital : cela changerait certes les quantités produites ${ }^{4}$, mais ne changerait rien au fait que, dans la nouvelle formule de répartition des revenus, les ressources resteraient utilisées au maximum possible permis par la technique. De la même façon, dans une société esclavagiste, le maître détient des droits sur une partie du produit du travail de l'esclave. Ici encore, si I'on affranchit les esclaves, et si on leur redonne leurs droits sur leur travail, il est probable que la nature de la production changera: on produira sans doute moins de biens de luxe, comme ceux que consommaient les maîtres esclavagistes, et plus de biens destinés à d'anciens esclaves de toute façon assez pauvres. Mais dans tous les cas, les ressources seront utilisées au mieux, compte tenu des règles plus ou moins arbitraires ainsi définies par la société sur l'attribution

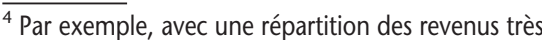
inégalitaire, on serait amené à produire 10000 Rolls Royce et 5 millions de bicyclettes. Avec une répartition plus égalitaire, on produirait 4 millions de voitures moyennes.
} 
des droits des acteurs de la vie économique sur les revenus de tel ou tel facteur ${ }^{5}$. Le modèle de Walras était purement conceptuel et son auteur $n^{\prime}$ avait pas imaginé qu'on pourrait un jour essayer de calculer effectivement un ordre de grandeur pour les solutions obtenues dans différents contextes. C'est pourquoi les premiers prolongements des travaux de cet économiste franco-suisse ${ }^{6}$ ont été d'abord théoriques. Mais la disponibilité, à partir du milieu du XX' ${ }^{\mathrm{e}}$ siècle, de moyens de calcul électroniques a donné lieu à des applications numériques, sous la forme des «modèles calculables d'équilibre général » Nous allons maintenant examiner ces deux aspects successivement.

\section{Les suites du modèle de Walras : prolongements théoriques}

L'analyse des aspects théoriques de l'équilibre général a été le fait d'une pléiade de Prix Nobel des années 1970, comme T. Koopman, R. Debreux ou K.J. Arrow. La conclusion essentielle de ces travaux est que les conditions les moins restrictives possibles qu'il faut accepter pour garantir l'existence d'un équilibre général comme l'envisageait Walras n'ont aucune chance d'être réunies en réalité. Cela tient au fonctionnement du marché : certes, si tous les producteurs égalisent le prix d'équilibre et le coût marginal, si tous les consommateurs maximisent leur utilité sous contrainte de budget, s'il existe des marchés d'assurance complètement concurrentiels pour n'importe quel aléas, et quelques autres conditions de ce genre, alors l'équilibre existe. Mais ce que I'on sait du fonctionnement des marchés permet $d^{\prime}$ 'assurer qu'en pratique ces conditions ne sont jamais réunies. Par exemple, les producteurs, au moment de la mise en production, ne savent pas quel sera le prix d'équilibre, et il n'existe pas de marchés sur lesquels il soit possible d'assurer les erreurs qu'ils pourraient

\footnotetext{
${ }^{5}$ Walras lui-même se définissait comme un « socialiste ». II souhaitait en particulier que les droits sur les terres soient attribués à l'État, et donc que toutes les terres soient nationalisées, l'État les louant chaque année aux agriculteurs susceptibles d'en faire un bon usage sur la base d'enchères. Son raisonnement s'applique non seulement aux terres mais à tout facteur de production dont la quantité est fixe et ne peut être modifiée par une action humaine.

${ }^{6}$ Léon Walras était français, et avait été formé à l'École des Mines. II chercha en vain un poste de professeur d'économie en France, et fut rejeté par I'Université. II finit par être reçu dans un concours de recrutement ouvert par le canton de Vaud en Suisse, ce qui fit de l'université de Lausanne un haut lieu de la science économique.
}

commettre de ce fait. De plus, il n'est pas sûr que si l'équilibre existe, il soit «stable » ${ }^{\text {. C'est }}$ pourquoi, en définitive, les travaux de haut niveau théorique sur l'équilibre général sont finalement beaucoup plus critiques vis-à-vis du " libéralisme » (dans sa forme dégradée standard) qu'on ne le croit généralement : il ne faut pas se laisser prendre à l'idée selon laquelle si les conditions d'un équilibre étaient réunies, alors, en effet, on tirerait le maximum des ressources disponibles.

Ces caveat sont cependant trop souvent ignorés des économistes praticiens, conseillers du Prince ou des organisations internationales. Ceux-là en sont restés à la version originale du modèle de Walras, qui présente l'avantage de pouvoir être résolue numériquement, si du moins on possède des données, on fait des hypothèses sur les fonctions algébriques, et on dispose d'une capacité de calcul convenable.

\section{Les modèles calculables d'équilibre général}

De fait, le modèle de Walras, dans les années 1860 , restait purement théorique et spéculatif : il était hors de question, à cette époque, de résoudre plusieurs dizaines de milliers d'équations avec un crayon et une gomme. II n'existait pas non plus de données crédibles pour cela - le tableau d'ensemble de Quesnay n'était plus à jour, et, du reste, il était encore bien trop sommaire. Les choses changent dès lors qu'on dispose d'ordinateurs et de «tableaux d'échange interindustriels ", sur le modèle de ceux que Wassily Léontief avait imaginé au début des années 1930, à l'origine, pour les besoins de la planification soviétique ${ }^{8}$.

\footnotetext{
${ }^{7}$ Un équilibre est stable si, quand on s'en écarte « un peu », il existe des forces susceptibles de ramener le système sur le point $d^{\prime}$ 'équilibre. C'est par exemple le cas du système composé d'une bille dans le fond $d^{\prime}$ une tasse : lorsqu'on remue la tasse, la bille s'écarte du fond, et monte sur les parois mais (pourvu que le choc sur la tasse n'ait pas été trop fort) elle est vite ramenée vers le fond, par la pesanteur et les forces de réaction sur les parois. Au contraire, une bille dont le centre de gravité est exactement au-dessus de la pointe d'un crayon est théoriquement «en équilibre ». Il est clair, en même temps, que l'état d'équilibre, ici, ne dure pas... il est « instable ».

${ }^{8}$ En dépit des apparences, les logiques du modèle de Walras et celle de la planification soviétique sont parentes : il s'agit dans les deux cas d'abord de tirer le maximum des ressources disponibles, ensuite de les répartir convenablement. Walras, du reste, n'a jamais pensé que la répartition «spontanée » des revenus issue du droit de propriété avait une quelconque « optimalité ». Tout au contraire, il militait pour la nationalisation des terres (afin de confisquer les rentes) et s'affichait « socialiste scientifique ».
}

De fait, les premiers modèles de ce type sont apparus au début des années 70 . On trouvera une abondante bibliographie sur les premières tentatives chez Shoven et Whalley [1] ou chez Robinson [2]. En France, Roland Courbis [3] fut un pionnier en créant à I'INSEE le modèle « Fifi » de l'économie française.

L'un des premiers modèles calculables d'équilibre mondial axé sur l'agriculture fut celui de I'IIASA à Vienne [4, 5]. À la même époque, un groupe d'Australiens [6] développait le modèle ORANI (qui n'était pas mondial à l'origine, mais qui reposait sur des données homogènes sur tous les principaux pays du monde). Dans les deux cas, il s'agissait de modèles d'équilibre général complets, avec un « zoom » sur l'agriculture, dont les activités étaient sensiblement plus détaillées que celles des autres secteurs.

Un peu plus tard, en s'appuyant au départ sur les données ORANI, mais cette fois organisées en une véritable matrice de comptes sociaux mondiale, Thomas Hertel développa à Purdue University un modèle bien plus général que celui de I'IIASA, connu sous le nom de GTAP ${ }^{9}$ [7]. Parallèlement, des modèles analogues étaient développés à la Banque mondiale et à I'OCDE, d'abord sous la forme d'une thèse modestement financée [8], puis avec une série de modèles plus formels [9], comme RUNS ${ }^{10}$ [10], vivement critiqué par Maurice Allais [11]. Plus tard encore, presque toutes les organisations internationales se sont dotées d'un instrument de ce type, comme la FAO et la CNUCED qui se sont unis pour créer le modèle ATSPM ${ }^{11}$. À la fin des années 70, on avait des données et des programmes, mais l'assemblage était laborieux, de sorte que l'utilisation des modèles exigeait des institutions de recherche lourdes, capable de gérer un important personnel d'informaticiens et de spécialistes des statistiques. Aujourd'hui, on dispose de programmes de calculs très faciles à employer, comme les nombreux « solvers » de GAMS ${ }^{12}$, qui permet-

${ }^{9}$ GTAP est I'acronyme de Global Trade Analysis Project. II s'agit en fait d'un énorme projet mené à I'université de Purdue, avec le double objectif de construire un modèle et de collecter les données nécessaires (qui peuvent être utilisées dans d'autres modèles). Cela n'a été possible que grâce à la collaboration de nombreux correspondants dans de nombreux pays.

${ }^{10}$ Modèle « Rural Urban North South ».

${ }^{11}$ Agricultural Trade Policy Simulation Model

${ }^{12}$ GAMS est I'acronyme de General Algebraical Modeling System. II s'agit en fait d'un langage de programmation, non d'un programme de résolution de systèmes d'équations. Cependant, ce langage est muni d'interfaces avec des «solvers » qui calculent les

solutions de systèmes d'équation ou de problèmes d'optimisation, comme par exemple les programmes 
Tableau 1. Quelques exemples d'évaluation des bénéfices de la libéralisation (en milliards de \$).

\begin{tabular}{|c|c|c|c|c|c|c|}
\hline \multirow[t]{2}{*}{ Source } & \multirow[t]{2}{*}{ Unité } & \multirow[t]{2}{*}{ Nature de l'expérimentation } & \multirow[t]{2}{*}{ Secteur concerné } & \multicolumn{3}{|c|}{ Région } \\
\hline & & & & $\begin{array}{l}\text { Gains } \\
\text { PVD }\end{array}$ & $\begin{array}{c}\text { Gains } \\
\text { Pays riches }\end{array}$ & $\begin{array}{l}\text { Total } \\
\text { Monde }\end{array}$ \\
\hline \multirow{4}{*}{$\begin{array}{l}\text { Banque mondiale } \\
\text { [15] }\end{array}$} & \multirow[t]{4}{*}{ US\$ 1997} & \multirow{4}{*}{$\begin{array}{c}\text { Abolition de toute barrière douanière en } 2015 \\
\text { (compte tenu de la croissance du PNB } \\
\text { entre } 1997 \text { et 2015) }\end{array}$} & Agriculture & 142 & 106 & 248 \\
\hline & & & Textiles & 24 & 17 & 41 \\
\hline & & & Total marchandises & 184 & 171 & 355 \\
\hline & & & Services & na & na & 668 \\
\hline \multirow[t]{3}{*}{ Brown et al. [16] } & \multirow[t]{3}{*}{ US \$1997 } & \multirow{3}{*}{$\begin{array}{l}\text { Baisse de } 33 \% \text { des tarifs post-Uruguay round, } \\
\text { sur biens et services, avec économies d'échelle }\end{array}$} & Agriculture & 10 & -13 & -3 \\
\hline & & & Manufactures & 50 & 113 & 163 \\
\hline & & & Services & 72 & 342 & 414 \\
\hline Brown et al. [17] & US \$1995 & Libéralisation totale, base 1995 & Tout & 370 & 1487 & 1857 \\
\hline Dessus et al. [18] & US $\$ 2000$ & Libéralisation totale & Tout & 274 & 62 & 337 \\
\hline Anderson et al. [19] & US \$1995 & Libéralisation totale pré-Uruguay Round & Tout & 108 & 146 & 254 \\
\hline Anderson et al. [20] & US $\$ 2003$ & Libéralisation Doha & Tout & 22 & 62 & 84 \\
\hline DFAT [21] & US\$ 1995 & Libéralisation totale, base 1995 & Tout & na & na & 750 \\
\hline \multirow[t]{4}{*}{ Hertel et al. [22] } & \multirow[t]{4}{*}{ US \$1995 } & \multirow[t]{4}{*}{ Baisse de $40 \%$ de la protection en 2005} & Agriculture & 26 & 43 & 69 \\
\hline & & & Manufactures & 58 & 12 & 59 \\
\hline & & & Transports & 6 & 16 & 22 \\
\hline & & & Autres & 10 & 223 & 333 \\
\hline Hertel et al. [23] & US $\$ 2000$ & Libéralisation totale & Tout & 22 & 62 & 84 \\
\hline Polanski [24] & US $\$ 2005$ & Libéralisation totale & Tout & 75 & 93 & 168 \\
\hline $\begin{array}{l}\text { Fontagné et } \\
\text { Ducreux [25] }\end{array}$ & US $\$ 2005$ & Libéralisation totale & Tout & 53 & 179 & 232 \\
\hline
\end{tabular}

Sources : Ackerman [12], Rollo [13] et Lipsicht [14].

tent de rechercher la ou les solutions (quand elles existent) de très grands systèmes d'équations simultanées non linéaires. On dispose aussi de bases de données comme celle qui avait été mise au point pour GTAP, et depuis constamment mise à jour. Il s'agit d'un ensemble organisé de «matrices de comptes sociaux » pour tous les pays du monde. II est extrêmement facile dans ces conditions de reproduire le modèle de Walras, en faisant quelques hypothèses accessoires sur les fonctions de production et les fonctions d'utilité. C'est à la portée de presque n'importe quel étudiant.

II n'est pas surprenant que, dans ces conditions, les modèles en question foisonnent. Dans les dernières réunions annuelles du consortium GTAP, plus de 300 modèles de ce type étaient présentés, certains " mondiaux », d'autres limités à une région ou un pays. La plupart d'entre eux ne présentaient que peu d'originalité. II ne faut donc pas s'étonner si, avec la même théorie, et les mêmes données, ils obtiennent des résultats très semblables. La

linéaires. Le langage de GAMS, élaboré par des informaticiens de la Banque mondiale dans les années 70, est spécialement adapté au traitement des données économiques, avec, en particulier, une gestion remarquable des ensembles et des sous-ensembles. Voir le site web: http://www.gams.com/docs/ intro.htm question, cependant, est de savoir dans quelle mesure le modèle walrassien standard est susceptible de répondre correctement aux questions que l'on se pose, en particulier celles qui concernent la libéralisation de l'agriculture. Nous allons d'abord examiner rapidement les principaux de ces résultats, avant de les discuter, puis de passer en revue les principales pistes qui s'ouvrent pour dépasser le cadre standard, et rendre ces modèles plus réalistes.

\section{Les résultats des modèles standard}

Pour étudier les effets possibles de la libéralisation avec les modèles dont il vient d'être question, la méthode est très simple: on commence par résoudre le modèle construit sur la base des politiques en vigueur actuellement. Puis on supprime toutes les mesures de politique (agricole ou non, ou certaines d'entre elles seulement, selon la nature de la réponse que l'on cherche), et on regarde comment se modifie la solution à la suite de cette suppression. En particulier, il est facile de calculer de cette façon les variations du PIB mondial. C'est ainsi que I'on peut évaluer les « bénéfices de la libéralisation ». Un échantillon de ces bénéfices figure dans le tableau 1. Les modèles qui sont listés dans la colonne de gauche diffèrent par les données sur lesquelles ils s'appuient, et par quelques points de détail ${ }^{13}$, mais ils sont tous essentiellement semblables au modèle walrassien de base.

Au premier abord, ces chiffres sont impressionnants. Même s'ils varient d'un modèle à l'autre, ils sont toujours positifs, et paraissent énormes. En réalité, ils ne sont pas si importants que cela : pour avoir une évaluation par tête, il faut diviser par l'effectif de la population mondiale, soit quelque chose qui est aussi de l'ordre de plusieurs milliards. Après cela, il ne reste plus au mieux que quelques dizaines de dollars par individu et par an: sûrement pas de quoi devoir considérer qu'il s'agit là de quelque

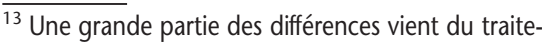
ment du capital et de la main-d'œuvre : selon que ces facteurs de production sont considérés comme fixes au niveau de chaque activité de production, ou bien peuvent être transférés sans coût d'un secteur à l'autre, les bénéfices de la libéralisation sont évidemment différents, et bien plus faibles en cas de fixité «forte » des facteurs : en même temps, il est bien clair qu'on ne peut instantanément transformer un agriculteur en médecin, ou un parc de charrues en centrale électrique... D'autres différences concernent par exemple la prise en compte de la « concurrence imparfaite ", et du fait que certains acteurs planifient leur production en tenant compte de ce que feront les autres (encore que ce comportement, s'il est très plausible dans un oligopole comme par exemple l'industrie automobile, soit sans doute très problématique dans le cas de l'agriculture).
} 
chose de nature à changer le visage de la planète!

En outre, ces quelques dizaines de dollars par tête sont fort mal réparties : les pays développés en gagnent l'essentiel. À l'intérieur même des pays en voie de développement, rien ne dit que ce ne seront pas les riches qui bénéficieront le plus des mesures de libéralisation : dans ce cas, la prétendue contribution de la libéralisation à l'éradication de la pauvreté serait un pur mensonge. Enfin, le fait que les gains soient toujours positifs ne devrait pas surprendre: toutefois, ce dernier point mérite quelques explications.

De fait, par construction, un modèle basé sur la théorie walrassienne ne peut en aucun cas donner un résultat négatif pour la libéralisation. Cela tient à ce qu'un tel modèle est en fait un " optimiseur » : pour un état donné de la technique, et étant donné les droits que chaque ménage possède sur les revenus des facteurs, on cherche le « point selle » qui correspond à la fois à la maximisation du profit des entrepreneurs et à la maximisation de l'utilité des consommateurs. II est bien connu que dans tout problème de maximisation l'addition d'une contrainte, au mieux, laisse la solution inchangée (si la contrainte n'est pas effective), mais normalement, abaisse la valeur du maximum (ou élève celle du minimum). En l'espèce, les droits de douane, ou les mesures restrictives du commerce, ajoutent des contraintes aux possibilités de production ou d'échange. II est donc naturel que les solutions «libérales" soient toujours globalement meilleures que les solutions " avec restrictions aux échanges ».

La question qui se pose à ce stade est celle de la vraisemblance de ce résultat. On retombe ici sur le problème qui avait été signalé plus haut à propos des théories physiocratiques : évidemment, si les marchés fonctionnaient bien, un système entièrement libéral serait préférable à tout autre. Mais l'expérience montre que ce n'est pas toujours le cas. Les plus dogmatiques des libéraux, comme Turgot, en ont fait I'amère expérience au XVIII ${ }^{\mathrm{e}}$ siècle. D'autres en ont souffert au moment de la "grande crise » des années 30, époque à laquelle furent mises en place les politiques agricoles que nous connaissons aujourd' hui $^{14}$

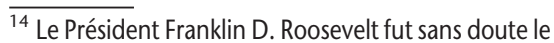
premier chef d'Etat à avoir délibérément et consciemment déconnecté l'agriculture du marché. II fut progressivement convaincu de la justesse de cette décision (qu'il n'avait pas prise volontiers !) par l'évolution de l'agriculture américaine au lendemain du premier Agricultural Adjustement Act. II est important de remarquer que, accusé à cette occasion de "socialisme", il se défendit en expliquant que l'agriculture était un secteur différent des autres, où le marché ne fonctionnait pas. II était donc un
}

En vérité, si les marchés fonctionnaient bien, il $n^{\prime} y$ aurait pas besoin d'économistes, car le monde serait tout à fait harmonieux, et aucune décision de politique économique ne serait nécessaire. Le seul problème qui se poserait alors serait un problème d'équité, celui des droits à attribuer à chacun sur les facteurs de production. Ce serait sans nul doute une difficulté : la notion de « justice » en économie ne va pas de soi. Du moins ne serait-il pas nécessaire que le gouvernement intervienne sans cesse dans la vie économique. II n'y aurait même pas besoin de firmes, comme le remarque Coase [28] : la firme est une institution bureaucratique, rendue nécessaire, justement, par le fait que la coordination entre les personnes qui y travaillent exige des canaux de circulation de l'information plus précis et plus contraignants que ceux qui sont constitués par les seuls rapports de prix. Ces réflexions conduisent à reconsidérer aussi bien la question de la libéralisation que celle des modèles destinés à en calculer les bénéfices.

Sur la libéralisation, il faut admettre que la nécessité de corriger les effets de l'insuffisance des mécanismes de marché exige aussi que l'on puisse se départir du respect aveugle des prix de marchés à un instant donné. Dans tous les pays, un des moyens les plus efficaces pour inciter les agriculteurs à investir et à augmenter leur production consiste à leur garantir les prix sur un horizon de temps assez long. C'est ces politiques de garantie de prix qui, en permettant aux agriculteurs d'emprunter et aux banques de prêter, ont permis la montée en puissance des agricultures américaines et européennes au cours des soixante dernières années, transformant la crainte de la pénurie en cauchemar des excédents. On a sûrement tort de continuer à produire plus qu'il ne faut, ce qui est un absurde gaspillage de ressources. Mais il vaut sûrement mieux crouler sous les excédents que souffrir de la famine, ce qui arriverait en l'absence de garanties de prix, comme on le voit en Afrique.

On pourrait sans nul doute pratiquer ces mêmes politiques de garantie de prix agricoles dans la plupart des pays en développement. On obtiendrait probablement de la sorte le même genre d'effets que ceux que l'on a constaté en Europe et aux États-Unis : un accroissement de la production agricole, une baisse de la population active agricole (qui se reconvertirait dans les autres activités), une importante substitution du capital au travail dans la production agricole (avec un capital largement financé par l'emprunt, devenu possible grâce à

vigoureux partisan de l'économie de marché, sauf dans des cas particuliers, dont l'agriculture faisait partie. Voir Lindsey [26] ou Galbraith [27]. la sécurité des prix), l'ouverture d'un marché rural important pour le secteur industriel local, en bref, une réelle amorce de développement. Mais on ne peut pas le faire, parce qu'il faudrait pour cela prévoir une régulation des importations qui ne passerait pas par le marché, et que cela est contraire aux dogmes du libéralisme. Bien entendu, pour apprécier sur des modèles I'utilité d'une telle politique, il faudrait que ceux-ci soient construits de façon à refléter les points cruciaux de l'analyse précédente. II nous faut donc maintenant expliciter ces points, avant d'examiner comment modifier les modèles pour en tenir compte.

\section{Les impasses du modèle walrassien}

Le point crucial de l'analyse précédente, c'est le rôle du risque dans les décisions des agriculteurs, en particulier des agriculteurs pauvres des pays en voie de développement, spécialement en rapport avec l'accumulation du capital. De fait, la raison profonde pour laquelle les agricultures des pays développés sont si « performantes " (un agriculteur suffit pour produire assez de nourriture pour une centaine de personnes, alors que les agricultures traditionnelles des pays en voie de développement parviennent tout au plus à nourrir 5 à 10 personnes), c'est que la quantité de capital par hectare y est considérable, qu'il s'agisse du capital privé, tel que tracteur ou pesticides, ou du capital public sous la forme d'infrastructures diverses.

Cela tient à ce que ces systèmes agricoles des pays développés ont pu pratiquer l'accumulation de ce capital, achetant progressivement des moyens de traction animaux, puis mécaniques, et augmentant leurs doses d'engrais et de pesticides année après année. $C^{\prime}$ est grâce à ces énormes investissements qu'il est possible de rémunérer plutôt bien la main-d'œuvre agricole (par rapport à ce qu'elle est rémunérée dans les pays sous-développés) tout en maintenant les dépenses alimentaires par tête dans les pays développés à un niveau plus bas qu'il n'a jamais été.

En général, les agriculteurs en question n'avaient pas les moyens de financer par euxmêmes les investissements en cause. En Europe comme aux USA, les agriculteurs étaient fort pauvres au début du $X X^{\mathrm{e}}$ siècle. Ils $n^{\prime}$ auraient pas pu investir autant $s^{\prime}$ ils n'avaient pas épargné. Proche du minimum vital, ils ne pouvaient guère épargner a priori, n'investissant qu'après avoir accumulé assez de fonds pour payer les biens capitaux qu'ils projetaient d'acheter. En général, ils épargnaient a posteriori en remboursant les emprunts qu'ils avaient contractés sur les produits de leurs ventes. Ils bénéficiaient ainsi d'un puissant « effet de levier » sur leur pauvre épargne personnelle. 
Or emprunter n'est possible que dans la mesure où l'on a des chances raisonnables de pouvoir rembourser. C'est ici qu'intervient I'incertitude sur, en particulier, les prix. Les prix agricoles sont fluctuants, cela est bien connu. Quand ils changent du simple au double en quelques mois, il est impossible d'admettre que le phénomène reflète une variation correspondante du coût marginal du produit en cause. Ce coût varie, certes, au fil du temps, au fur et à mesure du progrès technique et de l'évolution de la société, mais ces variations sont lentes et progressives. Cela n'a rien à voir avec les fluctuations des prix agricoles sur les marchés libres, qui varient dans de très fortes proportions de façon très soudaine. Or, si les prix varient beaucoup - en particulier, s'ils baissent - il est clair que le remboursement des emprunts va poser des problèmes, tant à la banque qu'à l'emprunteur... Dans ces conditions, soumis à de fortes fluctuations de prix, les agriculteurs pauvres n'empruntent pas, et par conséquent, $n^{\prime}$ accumulent pas de capital. De la sorte, tout « progrès » est interdit au système, piégé dans le « cercle vicieux de la pauvreté ». $C^{\prime}$ est bien ce que l'on constate dans les pays en voie de développement qui font trop confiance aux mécanismes de marché ${ }^{15}$.

Un autre point important peu correctement analysé dans les modèles walrassiens est le rôle de l'État comme fournisseur de « bien publics » - donc, par construction, échappant dans une large mesure à la régulation par le marché.

De fait, dans les pays développés, les États ont depuis longtemps généreusement fourni des infrastructures lourdes, mises à la disposition de tout le monde, dans les zones rurales. La plus importante est sans doute constituée par les services d'enseignement, qui ont joué un grand rôle en instruisant les enfants d'agriculteurs des techniques du métier, mais, bien plus encore, en leur fournissant le bagage intellectuel nécessaire pour quitter l'agriculture et s'employer dans les autres activités que la baisse du coût de l'alimentation permettait par ailleurs d'entreprendre. Cependant, les services d'enseignement ne sont pas les seuls : il y a aussi beaucoup de biens capitaux tangibles (que I'on songe aux efforts d'électrification rurale chers au Président Queuille !), ou même de services publics vendus en dessous de leur coût (on pense par exemple à la poste : il est clair que s'il fallait faire payer la distribution du courrier " au coût marginal », il faudrait instituer des tarifs dissuasifs pour les zones rurales).

Ces investissements dans les « infrastructures » représentent des sommes considérables, qui échappent dans une large mesure au marché,

\footnotetext{
$\overline{15}$ Les autres - tels que la Corée du Sud ou Taïwan sont souvent devenus développés...
}

et jouent évidemment un rôle crucial justement pour les petits agriculteurs isolés ${ }^{16}$. Or, ils sont très mal analysés dans les modèles de type walrassien, dans lesquels ils ne sont pris en compte que par l'intermédiaire des caractéristiques des fonctions de production (évidemment, la fonction de production du blé n'est pas la même selon que la livraison de la récolte se fait par un chemin goudronné ouvert en permanence ou par une route en terre inondée pendant la saison des pluies...). Or, il est très rare que soient mises en relation les dépenses publiques et les caractéristiques des fonctions de production. Cela fait apparaître à tort les dépenses publiques comme des dépenses «parasites", alors qu'elles sont la condition sine qua non de l'efficacité du secteur privé. Ces deux actions, l'accumulation du capital privé d'un côté, la fourniture de capital public de l'autre, sont au cœur du développement et sont ignorées du modèle walrassien. Cela tient pour l'essentiel de ce qu'il s'agit là de problèmes dynamiques, alors que le modèle walrassien est fondamentalement statique. Si I'on veut comprendre les effets de la libéralisation, il est essentiel de construire des modèles qui tiennent compte explicitement des remarques précédentes. En particulier, il est essentiel $\mathrm{d}^{\prime}$ aborder le problème sous un angle dynamique.

\section{La nécessité et la difficulté de rendre le modèle walrassien dynamique}

Depuis les efforts de Wassily Léontief, il serait injuste de dire qu'il n'y a eu aucune tentative pour «dynamiser » le modèle walrassien. La première idée qui vient à l'esprit dans ce domaine consiste à prendre en considération l'équation fondamentale du capital : le capital de l'année $t$ est égal au capital de l'année $t-1$, moins la dépréciation, plus l'épargne investie. Tous les MCEG comportent une fonction d'épargne (laquelle est évidemment complémentaire de la fonction de consommation). Tous ont un stock de capital. II n'est pas difficile de relier le stock de capital de l'année $t$, son taux de dépréciation, et le niveau d'épargne, pour obtenir le stock de capital de l'année $t+1$. Le modèle peut alors fonctionner de façon "récursive », la solution de l'année $t$ permettant le calcul de la solution de l'année $t+1$. On a ainsi l'illusion d'avoir un système dynamique.

\footnotetext{
${ }^{16}$ Voir par exemple lanchovichina et Kacker [29] qui trouvent que «pour le pays en voie de développement moyen, les plus grands dividendes de croissance proviennent d'abord des infrastructures publiques, suivi par les écoles secondaires, puis l'ouverture au marché et la consolidation financière ».
}

Les choses en réalité sont infiniment plus compliquées. Une première difficulté vient de ce que « le capital » n'est pas un objet homogène. On ne peut pas transformer instantanément une moissonneuse-batteuse en générateur électrique... Cela pose le problème de la spécificité du stock de capital de chaque secteur : à l'évidence, le capital qui existe dans l'agriculture ne peut pas être utilisé dans les industries mécaniques. II faut donc distinguer au moins autant de types de capital qu'il existe « d'industries» dans le modèle. Seul le «nouveau» capital peut s'investir dans un secteur ou dans un autre... Mais alors se pose le problème de savoir sur quelle base va se faire l'allocation du nouveau capital aux différents secteurs.

Évidemment, il est tentant de supposer que l'épargne nouvelle va s'investir dans les secteurs les plus "rentables». Mais comme il existe toujours un secteur plus rentable que les autres, cela implique que chaque année on n'investisse que dans un seul secteur... ce n'est pas conforme à la réalité, et cela donnerait aux séries chronologiques tirées du modèle une allure «bang-bang» du plus mauvais effet. Pour cette raison, ceux des MCEG qui prennent soin de considérer des stocks de capital différents pour chaque secteur procèdent à l'allocation de l'épargne entre les secteurs à l'aide de fonctions qui ressemblent à des fonctions de consommation, avec des élasticités de la « demande » d'investissement par rapport au prix (en l'espèce, la rentabilité du capital dans le secteur considéré).

Mais ce procédé ne tient pas compte d'un phénomène majeur en matière d'investissement : le décideur qui investit ne peut jamais savoir si le résultat sera conforme à ce qu'il espérait. II y a toujours un risque dans toute opération d'investissement, et cela explique du reste une quantité de phénomènes qui, autrement, seraient incompréhensibles. En particulier, si l'on attache un peu d'importance à toutes les théories financières sur la diversification des portefeuilles, il est naturel que les investisseurs «éparpillent » leurs investissements entre des opportunités qui n'ont ni la même espérance de gain, ni les mêmes caractéristiques en ce qui concerne les risques qu'ils présentent. Avec ce raisonnement, on est donc naturellement amené à lier l'allocation des nouveaux investissements entre les secteurs à des considérations de risque.

Il y a une autre raison de le faire : même sans faire intervenir les décisions d'investissement, à partir du moment où l'on se préoccupe du temps qui passe, il faut faire intervenir les délais de production: le temps qui s'écoule entre la décision de produire et le moment de la mise du produit sur le marché. Ceci implique aussi que les décisions de production ne soient pas prises sur la base des prix d'équilibre observés, 
mais sur la base $d^{\prime}$ 'anticipations auxquelles il est naturel d'associer un risque de non-réalisation. De la sorte, les considérations de risque vont jouer un rôle majeur dans les résultats des modèles - un point complètement ignoré des modèles walrassiens standard. II est tout spécialement important de remarquer que cette question du risque est cruciale pour expliquer les comportements des banques et, par conséquent, les rapports entre la politique monétaire et la production réelle.

Ces risques eux-mêmes ont des origines diverses : aléas météorologiques ou épidémiologiques «naturels» (contre lesquels il est au moins théoriquement possible de s'assurer), ou volatilité des prix, qui n'a rien de naturel, si bien qu'aucun mécanisme d'assurance basé sur la loi des grands nombres ne peut y remédier : les fluctuations de prix sont pour l'essentiel engendrées par l'instabilité de l'équilibre de n'importe quel marché dont la demande est rigide et I'offre élastique à court terme. Dans de tels marchés, en quelque sorte par construction, l'assurance est impossible : toute compagnie qui se risquerait à proposer un contrat d'assurance prix ne pourrait que courir à la faillite $[30,31]$. II faut donc trouver autre chose. C'est ce qui avait été fait dans les pays qui avaient mis en place des politiques de stabilisation ou de garantie de prix : de telles politiques sont maintenant interdites. Avant de les supprimer, il eut été souhaitable d'en évaluer l'utilité. Mais il aurait fallu pour cela utiliser des modèles susceptibles d'en faire apparaître l'utilité, au lieu du modèle walrassien, qui nie l'existence même du problème.

De fait, dans un modèle walrassien, les politiques monétaires et les politiques de stabilisation de prix des matières premières n'ont aucun objet. Toute intervention de l'État dans ce domaine ne peut que diminuer le bien-être global - dans le meilleur des cas, le laisser inchangé. Une telle conclusion est parfaitement contraire à l'expérience. Historiquement, les États sont intervenus, spécialement dans le domaine agricole, pour faire face à des dysfonctionnements majeurs des équilibres de marché.

Naturellement, les considérations précédentes jouent un rôle majeur dans le déterminisme de la production d'un État. Elles jouent encore plus lorsqu'il s'agit de représenter les flux d'investissements internationaux, qui dépendent non seulement des fluctuations de prix internes, mais encore des taux de change et des politiques monétaires. Or, ces flux d'investissements internationaux sont au cœur du processus de développement. C'est pourquoi la prise ne compte de ce genre de phénomènes est sûrement l'un des grands chantiers des modèles calculables d'équilibre général dans les prochaines années. Le modèle $I D^{3}$ du Cirad (voir l'article de Gérard et Piketty dans ce numéro) constitue un pas important dans cette direction. II donne des résultats très différents des autres, et tend à montrer que les résultats de la libéralisation seront plutôt négatifs. II serait évidemment souhaitable d'aller beaucoup plus loin dans cette direction.

\section{RÉFÉRENCES}

1. SHOVEN JB, WHALLEY J. Applied General Equilibrium Models of Taxation and International Trade : An Introduction and Survey. I Econ Lit $1984 ; 22$ : 1007-51.

2. ROBINSON S. Multisector Models of Developing Countries: A Survey. In : Chenery HB, Srinivasan TN, eds. Handbook of Development Economics. Amsterdam : North Holland, 1989 : 906-32.

3. COURBIS R, AGLIETTA M. Un outil pour le Plan: le modèle phiphi. Economie et statistique Mai. 1968.

4. FISCHER G, FROHBERG F, KEYSER MA, PARIKH KS. Linked national models : A tool for international food policy analysis. Amsterdam : Kluwer, 1988.

5. PARIKH KS, FISCHER G. Frohberg K, Guldbransen 0 . Toward free trade in agriculture. Amsterdam : Martinus Njhoff, 1988.

6. DIXON PB, PARMENTER BR, SUTTON J, VINCENT DP. ORANI : A multisectorial model for the australian economy. Amsterdam : North Holland, 1980.

7. HERTEL T, et al. Global trade analysis. Cambridge : Cambridge University press, 1997.

8. BURNIAUX J-M. Le radeau de la Méduse. Paris : Economica, 1988.

9. GOLDIN I, KNUDSEN O. Agricultural trade liberalization. Paris : OCDE, 1990.

10. BURNIAUX JM, VAN MENSBRUGGHE. Trade policy in a global context : technical specification of the Rural/Urban - North/South applical general equilibrium model. Paris: OECD Development Center Technical, paper 48, 1991.

11. ALLAIS M. Un libre échangisme suicidaire. Le Figaro, 5 juillet. 1993 ; (Paris).

12. ACKERMAN F. The shrinking gains from trade : a critical assessment of Doha Round Projections. Global Development and Environment Institute Working paper 05-01, Tuft University, Medford, USA, 2005.
13. ROLLO J. Consumer and multilateral trade liberalisation. Discussion paper. Brighton : The Sussex European Institute, 2006.

14. LIPSICHT A. Comparaison des études économiques portant sur l'impact du cycle de Doha. miméo DGTPE, Service des affaires multilatérales et du développement, juin 2006.

15. BANQUE MONDIALE. Global Economic Prospects and the Developing Countries, Washington. 2001.

16. BROWN DK, DEARDOFF AV, STERN RM. Computational Analysis of Multilateral Trade Liberalization in the Uruguay Round and Doha Development Round'. Research Seminar In International Economics Discussion Paper No. 489 School Of Public Policy. Ann Arbor: The University Of Michigan, 2002.

17. BROWN DK, DEARDOFF AV, STERN RM. CGE Modeling and Analysis of Multilateral and Regional Negotiating Options. " Research Seminar in International Economics Discussion Paper No. 468. Ann Arbor: School of Public Policy, The University of Michigan, 2001.

18. DESSUS SK, FUKAZAKU, SAADI R. La liberalisation multilatérale des droits de douanes et les pays en développement. Cah politique économique du centre de développement de I'OCDE $2001 ; 18 ; 38$ pp.

19. ANDERSON K, FRANCOIS I, HERTEL T, HOEKMAN B, MARTIN W. Potential gains from trade reform in the new millennium. Paper prepared for the $3 \mathrm{~d}$ global trade analysis conference. Mt Eliza (USA) : Monash University, 2000.

20. ANDERSON K, MARTIN W. Agricultural trade reform and the Doha development agenda. Washington DC : World Bank, 2005.

21. DFAT (DEPARTMENT OF FOREIGN AFFAIRS AND TRADE). Global Trade Reform: Maintaining Momentum. Canberra : Australia, 1999.

22. HERTEL TW, ANDERSON K, FRANCOIS JF, MARTIN W. 'Agriculture And Non-Agricultural Liberalization In The Millennium Round' Policy Discussion Paper No. 0016. Centre for International Economic Studies University of Adelaide, 2000.

23. HERTEL T, KEENEY R. What's at stake : the relative importance of export barriers, export subsidies and domestic support. In : Anderson K, Martin W, eds. Agricultural trade reform and the Doha agenda World Bank. Washington DC. 2005.

24. POLASKI S. Winners and Losers: Impact of the Doha Round on Developing Countries. Washington: Carnegie Endowment for International Peace, 2006. 
25. FONTAGNE L, DUCREUXY. A quantitative assessment of the Outcome of the Doha Development Agenda. Paris: Document de travail 10/2006 CEPII, 2006.

26. LINDSEY EK. The Roosevelt revolution first phase. Londres : Victor Gollancz, 1934.

27. GALBRAITH JK. The Age of Uncertainty. G. K. Hall Boston, 1977.

28. COASE RH. The Nature of the Firm. Economica $1937 ; 4$ : 386-405.
29. IANCHOVICHINA E, POOJA KACKER P. Growth Trends in the developing world : country forecasts and determinants World Bank Research working paper 3775, November 2005.

30. BOUSSARD JM. When risk generates chaos. Economic Behaviour Organization 1996; $29(96 / 05)$ : 433-46.

31. BOUSSARD JM. Price risk management in agricultural and other unstable markets ICFAI Journal of Risk and Insurance. 2006.

\section{Pour en savoir plus :}

FRANCOIS J. The Next WTO Round: North- South Stakes in New Market Access Negotiations. Centre for International Economic Studies, University of Adelaide, 2001.

FOLMER C, KEYSER MA, et al. The Common Agricultural Policy and the Mc Sharry Reform. Amsterdam : Elsevier, 1995. 